\title{
Chamoiseau: Nomadismes et intranquillité, «Présence Francophone» ", Revue internationale de langue et de littérature, n. 81
}

\section{Elena Pessini}

\author{
(2) OpenEdition \\ Journals \\ Édition électronique \\ URL : http://journals.openedition.org/studifrancesi/1501 \\ DOI : 10.4000/studifrancesi. 1501 \\ ISSN : 2421-5856 \\ Éditeur \\ Rosenberg \& Sellier
}

Édition imprimée

Date de publication : 1 décembre 2015

Pagination : 646-647

ISSN : 0039-2944

\section{Référence électronique}

Elena Pessini, «Chamoiseau: Nomadismes et intranquillité, «Présence Francophone» », Revue internationale de langue et de littérature, n. 81 », Studi Francesi [En ligne], 177 (LIX | III) | 2015, mis en ligne le 01 décembre 2015, consulté le 09 janvier 2021. URL : http://journals.openedition.org/ studifrancesi/1501; DOI : https://doi.org/10.4000/studifrancesi.1501

Ce document a été généré automatiquement le 9 janvier 2021.

\section{cc) (†) $\odot$}

Studi Francesi è distribuita con Licenza Creative Commons Attribuzione - Non commerciale - Non opere derivate 4.0 Internazionale. 
Chamoiseau: Nomadismes et intranquillité, «Présence Francophone» ", Revue internationale de langue et de littérature, n. 81

\author{
Elena Pessini
}

\title{
RÉFÉRENCE
}

Chamoiseau: Nomadismes et intranquillité, «Présence Francophone», Revue internationale de langue et de littérature, n. 81, 2014, pp. 181.

Dans son numéro 81, la revue «Présence Francophone» consacre son dossier à l'œuvre de Patrick Chamoiseau; Célestin MONGA et Samia KASSAB-CHARFI en signent la présentation. La professeure de l'université de Tunis a précédemment publié en 2012 un texte intitulé Patrick Chamoiseau (Paris, Gallimard/Institut français), entièrement centré sur la production de l'écrivain martiniquais. Ces travaux qui se suivent montrent bien comment ce dernier constitue actuellement le cœur des recherches de KassabCharfi. Le titre du dossier explicite le fil conducteur qui relie les différentes réflexions soumises au lecteur. Le Nomadisme (soulignons le pluriel employé dans le titre) doit être considéré à la fois comme la condition des habitants des Caraibes, toutes les Caraibes, dans leur parcours historique et dans leur présent contemporain, la condition des grands personnages créés par l'auteur, la clef d'une esthétique et d'une poétique qui se veulent à la fois reflet et interprétation, problématisation et questionnement sur un monde, notre monde, devenu fluide, mouvant, difficile à appréhender. Dans son intervention qui ouvre le recueil, Samia KASSAB-CHARFI examine l'œuvre de Chamoiseau à travers le concept de «nomadisme circulaire» qui semble - c'est l'hypothèse qui est 
mise en place - agir comme un fil rouge, en particulier dans les derniers récits de l'écrivain Biblique des derniers gestes (2002), Les neuf consciences du malfini (2009) et L'Empreinte à Crusoé (2012). Un nomadisme circulaire, et c'est le point de départ du travail de réflexion, qui définit la géographie de la Caraïbe, le rapport à l'espace éclaté, interrompu, haché au sein des îles antillaises, qui définit aussi le rapport à l'Histoire et à la langue. Nous assistons à une mise en place à la fois d'un nomadisme circulaire vécu par les héros chamoisiens - en particulier Balthazar Bodule-Jules, protagoniste de Biblique des derniers gestes - mais aussi à un nomadisme circulaire de l'écriture romanesque qui s'exprime par «les dyspnées du récit, de la syntaxe et de ses rythmes, dyspnées et altérations qui ne sont pas sans évoquer les 'souffrances du jazz'» (p. 11) Le lecteur avisé reconnaît bien sûr dans cette idée d'un déplacement qui ne se fait plus sur le mode de la conquête, selon le schéma européen qui fait suivre à un lieu de départ, un lieu d'arrivée à soumettre, les analyses théoriques qu'Édouard Glissant a, entre autres, développées dans Poétique de la Relation (1990). Dans cette analyse des déplacements et des départs, des retours en arrière et du sur-place que font les personnages chamoisiens, l'étude de L'Empreinte à Crusoé acquiert une importance fondamentale - les autres auteurs de ce recueil y reviennent souvent. L'île sur laquelle a échoué le Robinson de Chamoiseau est le lieu d'un nomadisme mental où se met en place l'intranquillité du personnage. Le concept de nomadisme est aussi appliqué à la lecture de l'ouvrage Écrire en pays dominé (1997) où Chamoiseau livre les secrets de sa "sentimenthèque», des lectures qui ont nourri son écriture et sa pensée: «S'affranchissant de la référence univoque à un auteur unique, Chamoiseau 'ameute' le peuple diversel des écrivains, reconnaissant nommément sa dette intellectuelle à leur égard, savourant et célébrant leurs poétiques ou leurs combats» (p. 21). Dans le deuxième article, Géotropisme de Chamoiseau, nous plongeons avec Jean-Louis CORNILLE, dans le récit Biblique des derniers gestes, dans sa matière narrative qui, par son aspect foisonnant et emmêlé, rappelle la luxuriance de la végétation martiniquaise, en particulier de la mangrove, mais surtout se révèle être tissé d'une multitude de relations avec d'autres œuvres d'écrivains majeurs de la littérature mondiale comme Perse, García Márquez, Glissant, Césaire, etc... Au sein de cette richesse de références et de points de contact, Cornille trouve la trace du personnage de l'esclave révolté Benito Cereno, mis en scène par Herman Melville dans le récit homonyme. Les affinités et les ressemblances qui sont soulignées sont effectivement surprenantes et laissent entrevoir des réseaux intertextuels qui sont le propre des grandes narrations. La troisième contribution Archéologie du cachot de Lydie MOUDILENo s'attarde sur Un dimanche au cachot et approfondit les rapports entre écriture, prison et mémoire dans l'un des derniers romans de Chamoiseau. Le lecteur pourrait être surpris par la présence d'une étude sur un lieu aussi circonscrit et aussi limité au sein d'un recueil qui s'interroge sur les nomadismes mais en réalité le cachot dont le narrateur devra tirer la jeune fille qui s'y est enfermée, en refusant tout contact avec l'extérieur, est un vestige de l'époque esclavagiste, un des lieux antillais que Chamoiseau veut sortir de l'oubli en le superposant à tous les autres cachots de l'Histoire auxquels les esclaves ont été contraints. Éric ноРPENOT, dans son article Le miel de l'alphabet. L'autobiographie archipélique de Patrick Chamoiseau, renifleur d'existence s'intéresse en particulier au premier des trois volets de l'autobiographie de l'écrivain, Chemin d'école (1994). Il s'agit pour Hoppenot de montrer comment Chamoiseau opère une subversion de l'écriture autobiographique qui ne se limite pas à mettre en scène des souvenirs, à dire le passé, à fixer par l'écriture des événements qu'il faut sauver de l'oubli mais serait plutôt 
tournée vers l'avenir, un avenir où les anciennes oppositions qui charpentaient la société coloniale sont définitivement déclarées obsolètes. Dans Chemin d'école, Chamoiseau met en place une nouvelle chronologie, un nouveau temps, un nouveau rythme qui diffère considérablement des rythmes imposés par l'institution scolaire de la métropole et il offre au lecteur la saveur d'une nouvelle langue. Guillaume PUGEARD DE GUBERT travaille, lui aussi principalement sur deux œuvres qui sont maintes fois reprises et objet d'analyse dans ce recueil de travaux critiques. Dans La parole et ses impossibles sont examinés Un dimanche au cachot et L'Empreinte à Crusoé même si les renvois à d'autres récits sont présents. Deux romans pour exprimer trois impossibles: l'impossible à décrire, l'impossible à dire et l'impossible à exprimer qui constituent les titres des différents paragraphes de l'étude. Dans Un dimanche au cachot, Chamoiseau explicite la difficulté à décrire l'horreur de l'esclavage, symbolisé par ce lieu, ce trou noir où étaient enfermés les rebelles, ceux qui osaient s'enfuir. Il s'agit pour lui de trouver au-delà de cette obscurité totale, à partir de ce gouffre insondable, des traces de lumières, des pistes de clarté auxquelles l'écriture va donner forme et consistance. «L'œuvre de Chamoiseau est une liane tendue au-dessus d'un gouffre et d'un abîme: l'indescriptible obscurité du déshumain et l'indicible opacité du vivant. Elle repose sur ces deux impossibilités: nommer l'indescriptible du déshumain vomi par la cale et réciter l'indicible du vivant retrouvé. La parole se décline ainsi en hoquets et en traces, en cris et en chants» (p. 87). Avec L'Empreinte à Crusoé, l'écrivain se heurte à l'obscur de ce qui a été recouvert par une taie de silence, au cours des siècles; le défi est autre. Il s'agit de reprendre une histoire, celle de Crusoé, pour laquelle tout semble avoir déjà été dit depuis Defoe jusqu'à Tournier. Le récit de Chamoiseau s'insère dans les interstices laissés par les autres pères de Robinson. Cilas KEMEDJo, dans Généalogie de l'errance, revient sur les rapports, maintes fois examinés, entre les signataires d'Éloge de la créolité (Chamoiseau, Bernabé et Confiant) et les deux grands intellectuels martiniquais que sont Aimé Césaire et Édouard Glissant. L'enquête s'avère ici particulièrement intéressante car elle est menée à travers l'analyse de la représentation urbaine dans l'œuvre de Chamoiseau. Dans l'En-ville des romans Texaco et Chronique des sept misères, l'écrivain martiniquais suit la trace indiquée par Édouard Glissant d'une saisie du réel de la Martinique qui toutefois ne doit pas s'enfermer dans une vision limitée, excluant le monde alentour. C'est en particulier la mise en scène de personnages tels que les pacotilleuses qui assurent les échanges du petit commerce des îles de la Caraïbe qui contribuent à forger une vision archipélique. La dernière contribution, La condition postmétisse de l'économiste Célestin MONGA sort des sentiers battus de la littérature et ouvre sur des perspectives stimulantes. Monga y analyse l'évolution de la pensée théorique de Chamoiseau qui s'est sensiblement modifiée au fil des années. «Il a subrepticement faussé le pas à la créolité métisse, militante et minimaliste de ses débuts (créolité énoncée surtout par opposition à la négritude) pour embrasser une humanité bien plus large car infinie, postmétisse en quelque sorte» (p. 135). Cette condition postmétisse, dont Monga trace les grands traits, a définitivement dépassé les limites exiguës de la créolité; elle ne s'affirme pas seulement comme une transformation des théories chamoisiennes mais peut se lire aussi dans une œuvre qui change, dans une écriture qui cherche de nouvelles issues.

2 La richesse de ce numéro de «Présence Francophone» réside bien dans la diversité des approches à l'œuvre de Chamoiseau mais ces travaux ont aussi le mérite de montrer 
comment se pencher sur un grand écrivain convoque nécessairement des problématiques qui concernent la littérature de façon générale. 